\title{
Congratulations to Poster Contest Winners; TMS Member Named ANS Fellow
} \section{themagazine \\ Poster Awards from TMS2020}

$J O M$ would like to recognize and congratulate poster award recipients from the Recycling of Secondary, Byproduct Materials, and Energy Poster Session held at the TMS 2020 Annual Meeting \& Exhibition (TMS2020) in San Diego,
California, in March 2020. The session was sponsored by the TMS Extraction $\&$ Processing Division (EPD) and the Recycling and Environmental Technologies Committee. The awardees are:

EPD/Light Metals Division (LMD) Recycling and Environmental Technologies - Best Poster Undergraduate: "Residual Quarry Sludge Stabilization to Prefabricate Sustainable Building Elements," Andres Felippe Restrepo Ramirez, Nicolas Zapata Perez, Yhan Paul Arias Jaramillo, and Juan Carlos Ochoa Botero, Universidad Nacional de Colombia

Graduate: "Characterization of Wasted LEDs from Tubular Lamps Focused on Recycling Process by Hydrometallurgy," Rafeal Oliveira, Amilton Botelho Júnior, and Denise Espinosa, University of São Paulo

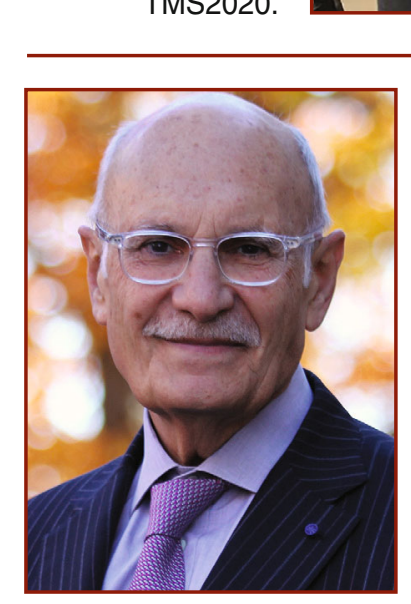

Diran Apelian

\section{Diran Apelian and ACRC Move to UCI}

Diran Apelian joined the University of California, Irvine, as the Distinguished Professor of Materials Science and Engineering in July 2019. He was previously the Alcoa Howmet Professor of Mechanical Engineering at Worcester Polytechnic Institute (WPI) for more than 30 years. The Advanced Casting Research Center (ACRC), an industry-university alliance initially founded to advance the use of light metals, followed Apelian to UCI in July 2020. According to UCI, the ACRC's relocation under

\section{member news}

Share the good news about your professional accomplishments! Contact Kaitlin Calva, JOM Magazine Managing Editor, at kcalva@tms.org. Please note that only news submitted by current TMS members will be considered.

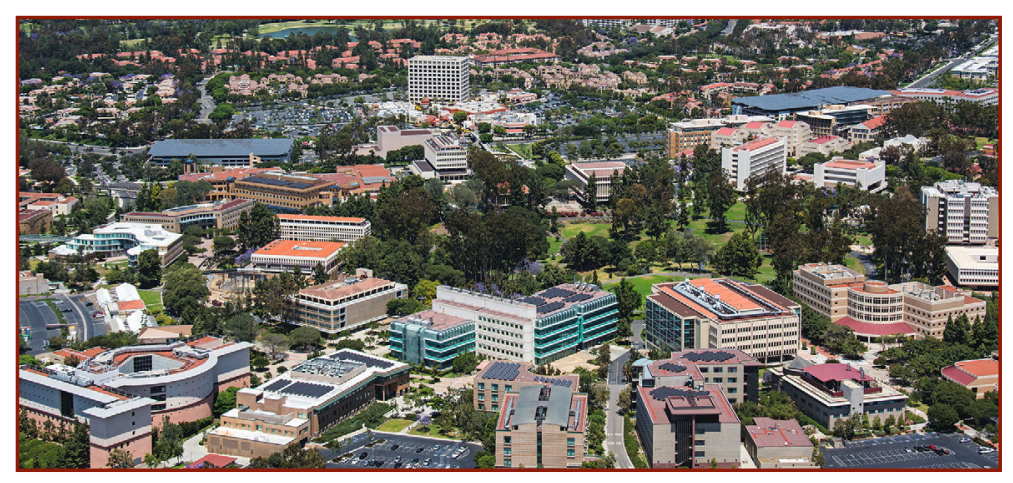

The University of California, Irvine, campus, new home to the ACRC. 


\section{TMS Member Receives ANS Fellow Award}

TMS member Kumar Sridharan, a professor in the Engineering Physics and Materials Science and Engineering Departments at the University of Wisconsin-Madison, was elected as a Fellow of the American Nuclear Society (ANS). He was awarded the honor during the 2020 ANS Virtual Annual Meeting in June along with eight other recipients. The distinction of Fellow is the highest membership grade honored by ANS. His citation reads, "for major research contributions in materials corrosion and degradation processes in nuclear energy systems, and for impact on the nuclear industry through the development of improved fuel cladding concepts as well as education and mentorship of undergraduate and graduate students."

Sridharan has authored more than 300 publications and has been a reserach mentor to more than 100 undergraduate and graduate students and postdoctoral reasearch assistants.

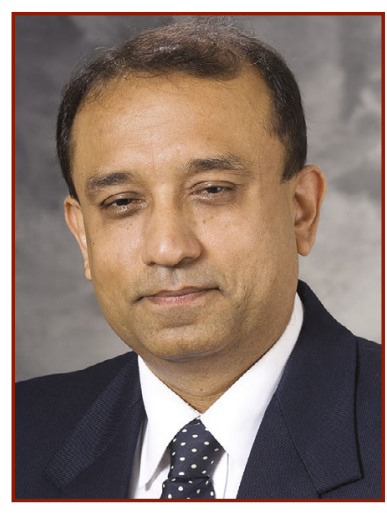

Kumar Sridharan

\section{TMS Welcomes New Members}

The TMS Board of Directors approved professional membership for the following individuals at its July 2020 meeting. Please join us in congratulating and welcoming them to all the privileges and benefits of TMS membership.

Abiodun, Yetunde; University of Lagos, Nigeria, Nigeria

Aghadavoudi Jolfaei, Mohsen; University of Warwick, United Kingdom

Allen, Stuart Keith; Mechatherm International Ltd., United Kingdom

Anand, Mohan; Warwick Manufacturing Group, United Kingdom

Anderson, Steve; Wagstaff Inc., United States

Awasthi, Shikha; Indian Institute of Science Bangalore, India

Barbosa, Isabella Tereza Ferro; Senac University Center, Brazil

Bennett, Tyler P.; Turbocam International, United States

Berg, Mike; Bruker Nano, United States

Bhandari, Geetanj; Rtec-Instruments Inc., United States

Bhargava, Parag; Indian Institute of Technology Bombay, India

Bhat Panemangalore, Devadas; National Institute of Technology Karnataka, India

Bibhanshu, Nitish; Oak Ridge National Laboratory, United States

Biener, Monika; United States

Boster, Connor; United States
Bowers, Mark; United Kingdom

Breguet, Jean-Marc; Alemnis AG, Switzerland

Bryan, Craig C.; Allied High Tech Products Inc., United States

Buswell, Deborah Jane; Australia

Cade, Austin; EAG Laboratories, United States

Carvalho Silva, Cleiton; UFC Campus Do Pici, Brazil

Chen, Shuonan; University of California, Riverside, United States

Claveau, Simon; STAS Inc., Canada

Cubillas Martinez, Fernando; Universidad de Sonora, Mexico

Dada, Modupeola; South Africa

Dahanayake, Vidumin; Anton Paar USA, United States

Drake, Robert; PROTO, United States

Du, Hui; MSE Supplies LLC, United States

Duque, Ramon; Altek LLC, United States

Essa, Khamis; University of Birmingham, United Kingdom

Ewasiuk, Rhonda J.; McSwain Engineering, United States

Farokhzadeh, Khorameh; Bruker, United States

Fernandez, Jorge; Altek LLC, United States
Friberg, Irma; United States

Ganguly, Partha; Baker Hughes, United States

Ghosh, Udayan; United States

Grauerholz, Blake; Fritsch Milling \& Sizing, Inc., United States

Greenwood, Anna; United Kingdom

Griessmann, Gunda; RHI Magnesita $\mathrm{GmbH}$, Austria

Guo, Xiaofeng; Washington State University, United States

Haertling, Carol; Los Alamos National Laboratory, United States

Hall, Steve; TA Instruments, United States

Hamilton, Bob; Precimeter Inc., United States

Heffernan, Michael; United Kingdom

Hernandez-Negrete, Ofelia; Universidad De Sonora, Mexico

Heslin, Edel; Taylor Francis Group, United Kingdom

Hetrick, Rob; Claudius Peters Projects $\mathrm{GmbH}$, United States

Hill, Tina; Bruker, United States

Hirsch, Edward A., Allied High Tech Products, United States

Hoover, Malini; Advanced Optical Technologies, United States 
Hostettler, Simon; Synton Mdp AG, Switzerland

Jackson, Daniel; Pyrotek Inc., United States

Jahangiri, Negin; Leica Microsystems, United States

Jencks, Danielle E.; Pratt \& Whitney, United States

Jetten, Peter; Pyrotek Inc., United States

Jiang, Xiaoping; MTI Corporation, United States

Johanns, Kurt; KLA Corporation, United States

Khosla, Tushar; Rtec-Instruments, Inc., United States

Kulkarni, Ajit; IIT Bombay, India

Lamar, Rick; Goodfellow Corp., United States

Lang, Melanie; Formalloy, United States

Lanza, Mark S.; Pacific Northwest National Laboratory, United States

Lau, Siu Chung; Hong Kong

Lavanya, S.; Indira Gandhi Center for Atomic Research, India

Lecroy, James; RCS, United States

Leosson, Kristjan; DT Equipment, Iceland

Leverette, Bobby; Pace Technologies, United States

Liddell, Keith; United Kingdom

Liu, Jian; Polaronyx Inc., United States

Lok, Jonathan; United States

Lopez Rodrigues, Isbelis; United Kingdom

Loukus, Josh; REL Inc., United States

Mahajanam, Sudhakar; Stress Engineering Services Inc., United States

Masi, Luca; Granta Design/Ansys Inc., United Kingdom

Mason, John; Solar Turbines Incorporated, United States

Mehta, Subal; Almex USA Inc., United States
Morel, Pierre; Rtec-Instruments,Inc., United States

Mouginot, Roman; Femtotools AG, Switzerland

Mu, Yunpeng; CIMM Group Co. Ltd., China

Muntwyler, Simon; Femtotools AG, Switzerland

Nair, Chandrasekharan; Intel Corporation, United States

Nalwa, Kanwar S.; Indian Institute of Technology Kanpur, India

Nedeljkovic, Dragutin; American University of the Middle East, Kuwait

Nisar, Ambreen; Florida International University, United States

Norris, Adam; Glama, Germany

Obali, Akin; Sistem Teknik Industrial Furnaces Ltd., Turkey

Olszta, Matt; Pacific Northwest National Laboratory, United States

Ortiz-Zavaleta, Gerardo; Innofacturing Solutions, United States

Parkes, Ken; Wagstaff Inc., United States

Pasini, Kellie; United States

Patel, Jitendra K.; United Kingdom

Patel, Chetan; United States

Pegg, Elise; United Kingdom

Potter, Megan; SCR Technologies, United States

Prakash, Chandra; Johns Hopkins University, United States

Ranz, Iris; United Kingdom

Rasovic, llija; United Kingdom

Reali, Jon C.; Magnitude 7 Metals, United States

Regmi, Abiral; United States

Rice, Christian; Nanovea, United States

Rui, Gao; Massachusetts Institute of Technology, United States

Schultz, Benjamin F;; Matsys Inc., United States

Seaman, Elyn; Hitachi High Technologies America Inc., United States

Shea, Ryan J.; Howmet Aerospace, United States
Shin, Dongwoon; University of Utah, United States

Shojaee, S. Ali; Thermo Fisher Scientific, United States

Singh, Sukhjinder; IPS Ceramics USA, United States

Sleep, Kyle; Limpact International Limited, Canada

Soellner, Wolfgang; Airbus Defence and Space, Germany

Stauffer, Terry E.; Microtrac, United States

Stiller, Adrian; Abo Akademi University, Finland

St-Onge, Dominic; Tekna, Canada

Szeszko, Justyna; Femtotools AG, Switzerland

Thompson, Taylor; Proto, United States

Tilak, Ravidra; Almex USA, United States

Tourountzis, Konstantinos; Alumil, Greece

Uguccioni, Paul; International Magnesium Association, United States

Valdis, Patrick; Nanovea, United States

Vichos, Peter; Netzsch Instruments, United States

Wagstaff Parkes, Barbara; Wagstaff Inc., United States

Walsh, Thomas; Tenova Inc., United States

Wang, Shuo; University of Maryland, College Park, United States

Wang, Yan; Georgia Institute of Technology, United States

Xiao, Jun; Rtec-Instruments Inc., United States

Yang, Menghao; University of Maryland, United States

Zhou, Fang; Carl Zeiss Microscopy LLC, United States

*Membership grade recommendations are based on a review of credentials provided by the individuals. These credentials are taken on the honor system and not independently verified except by exception. 